\title{
Education, Financial Stress, and Trajectory of Mental Health During the COVID-19 Pandemic
}

Yanping Jiang ${ }^{1}$, Samuele Zilioli ${ }^{1,2}$, Rhonda N. Balzarini ${ }^{3,4}$, Giulia Zoppolat ${ }^{5}$, Richard B. Slatcher ${ }^{6}$

\author{
${ }^{1}$ Department of Psychology, Wayne State University, Detroit, MI, United States \\ ${ }^{2}$ Department of Department of Family Medicine and Public Health Sciences, Wayne State \\ University, Detroit, MI, United States \\ ${ }^{3}$ Department of Psychology, Texas State University, San Marcos, TX, United States \\ ${ }^{4}$ The Kinsey Institute, Indiana University, Bloomington, IN, United States \\ ${ }^{5}$ Department of Experimental and Applied Psychology, Vrije Universiteit, Amsterdam, \\ Netherlands \\ ${ }^{6}$ Department of Psychology, University of Georgia, Athens, GA, United States
}

Correspondence concerning this article should be addressed to Yanping Jiang (yanping.jiang@wayne.edu). Department of Psychology, Wayne State University, 5057

Woodward Avenue, Detroit, Michigan 48202.

\section{Acknowledgement:}

The preparation of the manuscript was partly supported by a Faculty Competition for Postdoctoral Fellowship from Wayne State University (Samuele Zilioli). 


\begin{abstract}
Socioeconomic disparities in mental health have been reported during the COVID-19 pandemic. However, few studies have examined the mechanisms through which such disparities in mental health occurred. This pre-registered study aimed to examine socioeconomic disparities, as indexed by education levels, in the trajectory of mental health at the early stages of the COVID-19 pandemic and whether financial stress associated with the pandemic mediated socioeconomic disparities in mental health. Data were drawn from the Love in the Time of COVID project, of which we included four waves of data $(N=2,204)$ collected between March $27^{\text {th }}$ and June $21^{\text {st }}, 2020$. Education was assessed at baseline, and mental health outcomes (i.e., eudaimonic well-being, positive affect, negative affect, depressive and anxious symptoms) and financial stress associated with the COVID-19 pandemic were assessed at each wave. Results indicated that there were educational disparities in eudaimonic well-being, negative affect, and depressive and anxiety symptoms at baseline, with those with lower education levels reporting poorer mental health. However, education did not amplify disparities in mental health outcomes over time, showing no associations with the rates of change in mental health outcomes. Financial stress mediated the associations between education and eudaimonic well-being, negative affect, and depressive and anxious symptoms at baseline, and there were no temporal variations in the mediation effects of financial stress. These results highlight persistent educational disparities in mental health at the early stages of the COVID-19 pandemic, and such educational disparities may be partially explained by financial stress associated with the COVID-19 pandemic.
\end{abstract}

Keywords: COVID-19 pandemic; socioeconomic disparities; education; mental health; financial stress; depressive symptoms; anxiety. 


\section{Education, Financial Stress, and Trajectory of Mental Health During the COVID-19 \\ Pandemic}

The ongoing COVID-19 pandemic has dramatically changed individuals' lives across the world. As of April 30 ${ }^{\text {th }}, 2021$, the COVID-19 pandemic had resulted in more than 150 million individuals becoming infected and caused more than 3.1 million deaths worldwide (Center for Systems Science and Engineering at Johns Hopkins University, 2021). In addition to the tremendous illness and loss related to the COVID-19 infection, the COVID-19 pandemic puts many individuals under enduring stress (Cooke, Eirich, Racine, \& Madigan, 2020; Salari et al., 2020). The policies that were put in place to help reduce the spread of COVID-19-including lockdown and stay-at-home orders — not only disrupted individuals' daily routines but also led to spikes in unemployment (Faria-e-Castro, 2021; Kochhar, 2020; Pappas, 2020), putting many at risk of experiencing financial stress and strain (Coibion, Gorodnichenko, \& Weber, 2020; Crayne, 2020).

The stressors introduced over the course of the COVID-19 pandemic, including financial stress, may take a toll on individuals' mental health. Indeed, initial research showed that people experienced heightened levels of depression and anxiety and decreased levels of eudaimonic well-being (e.g., purpose in life) during the COVID-19 pandemic (Paleari, Pivetti, Galati, \& Fincham, 2021; Wilson et al., 2020). For example, evidence from a meta-analysis found that the presence of depression during the COVID-19 pandemic was about seven times greater than the prevalence of depression before the pandemic (25\% vs. 3.4\%, Bueno-Notivol et al., 2021). Findings from longitudinal studies have also shown that individuals may experience a prolonged deterioration in mental health during the pandemic (O'Connor et al., 2020). However, some studies suggest that individuals may exhibit resilience to the COVID-19 pandemic, showing 
improvements in mental health after the beginning of the COVID-19 lockdown (Daly, Sutin, \& Robinson, 2020; Fancourt, Steptoe, \& Bu, 2021; Wang et al., 2020). For example, a metaanalysis of longitudinal studies and natural experiments found that most individuals showed psychological resilience to the effects of the COVID-19 lockdown on mental health (Prati \& Mancini, 2021). Such resilience suggests that although people may have been initially impacted by the stressors brought on by the pandemic and have had mental health challenges because of it, not all have been affected to the same extent.

Notably, studies have shown that the impact of the COVID-19 pandemic on mental health is not uniform across all walks of life (Purtle, 2020). Some sociodemographic factors, including socioeconomic status (SES), have long been established as potent social determinants of mental and physical health outcomes (Adler \& Ostrove, 1999). Education- a key indicator of SES- seems to have played an important role during the COVID-19 pandemic, with implications for people's mental health (e.g., Fancourt et al., 2021; Prati \& Mancini, 2021). For example, a large cohort study in the UK found that individuals with lower education (i.e., secondary education or lower) were more likely to report higher levels of depression and anxiety at the start of the COVID-19 pandemic lockdown, though such educational disparities in mental health decreased over the 20-weeks after the start of the lockdown (Fancourt et al., 2021). To date, however, no studies have identified the potential psychological mechanisms through which education has contributed to mental health disparities during the COVID-19 pandemic. The identification of such mechanisms may shed light on the development of public health initiatives to reduce mental health disparities associated with the COVID-19 pandemic.

Psychological stress could be an important potential mechanism, given that stress has long been recognized to contribute to SES disparities in health (Baum, Garofalo, \& Yali, 1999). 
Recent studies, in particular, suggest the importance of testing SES-specific stress exposure (e.g., financial stress) in clarifying SES-health links (Cundiff, Boylan, \& Muscatell, 2020). Indeed, previous studies have reported a significant relationship between lower levels of education and higher levels of financial stress (e.g., Parnia \& Siddiqi, 2020; Rios \& Zautra, 2011). In addition to the elevated risk of exposure to psychological stress, individuals with lower education are also less likely to adapt to stress (Gallo \& Matthews, 2003), putting them at increased risk of experiencing poorer health outcomes following exposure to stressors (Jiang, Zilioli, RodriguezStanley, Peek, \& Cutchin, 2020). In the context of the COVID-19 pandemic, individuals with low education have been reported to be disproportionately affected by job loss due to the COVID-19 pandemic lockdown (Kochhar, 2020), which may contribute to elevated levels of financial stress among this population. Increased levels of financial stress associated with the COVID-19 pandemic, in turn, have been documented to be associated with poorer mental health outcomes, such as depression (Thayer \& Gildner, 2020). Few studies, however, have explicitly examined the potential mediation role of financial stress in contributing to educational disparities in mental health during the COVID-19 pandemic.

\section{The Current Study}

The current study aimed to examine the mediation effect of financial stress associated with the COVID-19 pandemic (hereafter referred to as financial stress) on the association between education and mental health. Specifically, mental health was assessed using multiple indicators, including eudaimonic well-being, positive affect, negative affect, and depressive and anxiety symptoms. We hypothesized that financial stress would mediate the associations between education and mental health outcomes. A secondary purpose of this study was to replicate previous studies on changes in individuals' mental health during the COVID-19 pandemic (e.g., 
Daly et al., 2020) by examining the trajectory of mental health at the early stages of the COVID19 pandemic (between March and June 2020) and the potential educational disparities in the trajectory of mental health. We hypothesized that participants would show mental health "recovery", exhibiting declines in mental health problems over time, and individuals with lower education would be less likely to recover than their counterparts with higher education. The hypotheses for this study were pre-registered on the Open Science Framework (OSF; https://osf.io/r2wsn).

\section{Methods}

\section{Participants and Procedure}

Participants were drawn from the first four waves of the Love in the Time of COVID study, an ongoing longitudinal project examining the effect of the COVID-19 pandemic on how people connect, relate, and cope during the pandemic. Participants were recruited from social media sites (e.g., Facebook), word of mouth, and the project website (https://loveinthetimeofcovid.me). Participants who were 18 years of age or older and agreed to participate in the study were asked to complete an online survey (baseline launched on March $\left.27^{\text {th }}, 2020\right)$ after the World Health Organization declared COVID-19 a global pandemic on March $11^{\text {th }}, 2020$. Participants were invited to complete follow-up surveys every two weeks. The survey was first available in English and then was translated into 10 different languages (Spanish, Turkish, Thai, Chinese, Dutch, French, German, Indonesian, Italian, and Portuguese) using back-translation (for details, see Colina, Marrone, Ingram, \& Sánchez, 2017; Tyupa, 2011). The survey consists of questionnaires about individuals' experiences since the onset of the COVID-19 pandemic, including financial stress and mental health (for a complete list of the measures and project protocol, see https://osf.io/yjkhx). Participation in this study was voluntary, 
and the study procedure was approved by the Institution Review Board at the University of Georgia.

A total of 5,575 adults initially participated in the project, of which 3,371 were dropped from the analyses because they did not pass the data quality check $(n=111)$ and did not provide data on outcome variables at baseline and at least one of the three follow-ups $(n=3,260)$. Data quality was assessed through two attention check questions and a single item asking participants how much attention they had paid to the survey. Participants were considered not to pass the quality check if they did not select the correct answer in the attention check questions or reported paying very little or no attention to the survey. This resulted in a final sample of 2,204 participants from 53 countries in the current analysis $(82.0 \%$ female, $69.9 \%$ having a bachelor's degree, Mage $=32.75 \pm 12.59$ years $)$.

\section{Measures}

Education. Education was assessed at T1 by asking participants' highest educational attainment. Responses options were $1=$ less than 6 years, $2=$ less than 12 years, $3=$ high school graduate, $4=$ some university, $5=$ associate degree, $6=$ university graduate, $7=$ master level degree, and $8=$ doctoral degree, with a high score reflecting a higher level of education.

Financial Stress. Financial stress in the past two weeks was assessed using a single item ("To what degree has the recent COVID-19 outbreak negatively impacted your financial situation") at baseline (T1), 2-week follow-up (T2), 4-week follow-up (T3), and 6-week followup (T4). This item was initially derived from Pearlin, Menaghan, Lieberman, and Mullan (1981) and revised by Okechukwu, El Ayadi, Tamers, Sabbath, and Berkman (2012). Responses options ranged from $1=$ not at all to $5=$ extremely, with a higher score reflecting a higher level of financial stress. 
Eudaimonic Well-Being. Two items (i.e., "I have been completely satisfied with my life", "My life has had a clear sense of purpose") were used to assess eudaimonic well-being in the past two weeks on a 7 -point scale from $1=$ not at all to $7=$ completely at each wave (Diener, Emmons, Larsen, \& Griffin, 1985). Responses on these two items were averaged to create a composite for eudaimonic well-being, with a higher score reflecting a higher level of eudaimonic well-being. The correlation coefficients between these two items were 0.62 at $\mathrm{T} 1,0.65$ at $\mathrm{T} 2$, 0.69 at $\mathrm{T} 3$, and 0.71 at $\mathrm{T} 4$.

Positive Affect. Four items (e.g., happy, excited) from the Positive and Negative Affect Scale (Watson, Clark, \& Tellegen, 1988) were used to assess positive affect in the past two weeks on a 5-point scale from $1=$ not at all to $5=$ extremely at each wave. Responses on the items were averaged to calculate a mean score for positive affect, with a higher score reflecting a higher level of positive affect. The Cronbach's alpha was 0.72 at T1, 0.73 at T2, 0.75 at T3, and 0.76 at $\mathrm{T} 4$.

Negative Affect. Negative affect was assessed using eight items from the Positive and Negative Affect Scale (Watson et al., 1988) at each wave. Participants were asked to report to what degree they felt in a certain way (e.g., bored, distressed) in the past two weeks on a 5-point scale from $1=$ not at all to $5=$ extremely. Responses were averaged to create a composite for negative affect, with a higher score reflecting a higher level of negative affect. The Cronbach's alpha was 0.84 at $\mathrm{T} 1,0.85$ at $\mathrm{T} 2$, and 0.86 at $\mathrm{T} 3$ and $\mathrm{T} 4$.

Depressive and Anxiety Symptoms. Three items (i.e., little interest, depressed, uneasy) from the Patient Health Questionnaire-4 were used to assess depressive and anxiety symptoms in the past two weeks on a 4-point scale from $1=$ not at all to $4=$ nearly every day (Löwe et al., 2010) at each wave. Given that the anxiety item was highly correlated with the two depression 
items at each wave $(r \mathrm{~s}=0.35-0.59)$, responses on these three items were averaged to calculate a mean score for depressive and anxiety symptoms, with a higher score reflecting more severe depressive and anxiety symptoms. The Cronbach's alpha was 0.76 at T1, 0.79 at T2, and 0.80 at T3, and 0.79 at T4.

Covariates. Participants were asked to report their age and gender (dummy coded as male vs. female; male vs. other gender) at baseline. Self-isolation behavior was also assessed using a single item to ask participants to what degree they had practiced self-isolation behavior (i.e., staying at home and avoiding public spaces) in the past two weeks on a 5-point scale from $1=$ not at all and $5=$ completely at each wave. These covariates were included in the analyses due to their associations with mental health associated with the COVID-19 pandemic reported in previous studies (Daly et al., 2020; Hwang, Rabheru, Peisah, Reichman, \& Ikeda, 2020).

\section{Statistical Analyses}

Multilevel modeling (MLM) was used to test the effects of education on mental health outcomes, of which education was included as a predictor at Level 2, and the changes of mental health outcomes over time within participants were modeled by including time as a predictor at Level 1. Time (weeks) was centered at baseline so that the intercept reflected mental health outcomes at baseline. The intercept and slope of mental health outcomes were randomly estimated at Level 2. Continuous variables at Level 2 were grand-mean centered (Enders \& Tofighi, 2007). MLMs were separately carried out for negative affect, positive affect, depressive and anxiety symptoms, and eudaimonic well-being. Multilevel mediation modeling (MMM) was performed to test the mediation effect of financial stress on the associations between education and mental health outcomes. Specifically, we performed the longitudinal 2-1-1 mediation model (see Equation 1) following the procedures proposed by Preacher, Zhang, and Zyphur (2011) and 
Berli, Inauen, Stadler, Scholz, and Shrout (2020), in which we estimated the effects of education on the intercept and changes (i.e., slope) of both financial stress and mental health outcomes. The indirect effect was tested using the product of between coefficients (i.e., $\beta_{\mathrm{m} 01} \times \beta_{\mathrm{y} 02} ; \beta_{\mathrm{m} 11} \times \beta_{\mathrm{y} 02}$ ), of which $\beta_{\mathrm{m} 01} \times \beta_{\mathrm{y} 02}$ reflects the indirect effect of financial stress at baseline, and $\beta_{\mathrm{m} 11} \times \beta_{\mathrm{y} 02}$ reflects temporal variations in the mediation effects of financial stress over time. Given that the bootstrapping method is not available for this specification, the delta method was used to test the significance of the indirect effect. All models were first carried out without covariates and controlled for covariates (i.e., gender, age, and self-isolation behavior) for the mediator (if applicable) and mental health outcomes. Gender and age were included as Level 2 predictors, and self-isolation behavior was included as a predictor at Level 1. The incidence of missing data was $23.9 \%$, and the full information maximum likelihood was used to handle missing data (Larsen, 2011). All analyses were performed using maximum likelihood with robust standard errors in Mplus 7.0.

Equation 1:

$$
\begin{aligned}
& \text { Financial stress } \mathrm{ti}_{\mathrm{i}}= \\
& \qquad \begin{aligned}
\mathrm{m} 0 \mathrm{i} & +\pi_{\mathrm{m} 1 \mathrm{i}}\left(\text { time }_{\mathrm{ti}}\right)+\varepsilon_{\mathrm{mti}} \\
& \pi_{\mathrm{m} 0 \mathrm{i}}=\beta_{\mathrm{m} 00}+\beta_{\mathrm{m} 01}\left(\text { education }_{\mathrm{i}}\right)+\mu_{\mathrm{m} 0 \mathrm{i}} \\
& \pi_{\mathrm{m} 1 \mathrm{i}}=\beta_{\mathrm{m} 10}+\beta_{\mathrm{m} 11}\left(\text { education }_{\mathrm{i}}\right)+\mu_{\mathrm{m} 1 \mathrm{i}}
\end{aligned}
\end{aligned}
$$

$$
\begin{aligned}
& \text { Mental health } \pi_{\mathrm{ti}}= \\
& \pi_{\mathrm{y} 0 \mathrm{i}}+\pi_{\mathrm{y} 1 \mathrm{i}}\left(\text { time }_{\mathrm{ti}}\right)+\pi_{\mathrm{y} 2 \mathrm{i}}\left(\text { financial stress }_{\mathrm{ti}}\right)+\varepsilon_{\mathrm{yti}} \\
& \pi_{\mathrm{y} 0 \mathrm{i}}=\beta_{\mathrm{y} 00}+\beta_{\mathrm{y} 01}(\text { education })+\beta_{\mathrm{y} 02}(\text { financial stress })+\mu_{\mathrm{y} 0 \mathrm{i}} \\
& \pi_{\mathrm{y} 1 \mathrm{i}}=\beta_{\mathrm{y} 10}+\beta_{\mathrm{y} 10}(\text { education })+\mu_{\mathrm{y} 1 \mathrm{i}} \\
& \pi_{\mathrm{y} 2 \mathrm{i}}=\beta_{\mathrm{y} 20}
\end{aligned}
$$

\section{Results}




\section{Descriptive Results}

Table 1 displays the characteristics of the sample. Table 2 displays the correlation coefficients between variables. Results showed that education was positively correlated with eudaimonic well-being but negatively correlated with negative affect and depressive and anxiety symptoms at each wave $(p s<.001)$. Education was not correlated with positive affect at any of the four waves ( $p s>.10)$. In addition, education was negatively correlated with financial stress at each wave $(p s<.001)$. Financial stress was negatively correlated with eudaimonic well-being ( $p$ s $<.01)$ but positively correlated with negative affect and depressive and anxiety symptoms ( $p s$ $<.001)$ at each wave. Financial stress was not correlated with positive affect, with exceptions that there were correlations between baseline financial stress and baseline positive affect $(r=-$ $0.05, p=.020)$ and between T1 financial stress and baseline and T1 positive affect $(r=-0.05, p$ $=.037 ; r=-0.05, p=.036$; respectively).

\section{[INSERT TABLE 1 HERE]}

[INSERT TABLE 2 HERE]

\section{Trajectory of Mental Health}

The unconditional MLM models showed that there were significant effects of time (i.e., average slope [rate of change]) on eudaimonic well-being $(b=0.035,95 \%$ CI [0.024, 0.045], $p$ $<.001)$ and positive affect $(b=0.008,95 \%$ CI $[0.003,0.014], p=.004)$, indicating that participants exhibited an increase in eudaimonic well-being and positive affect over time. Time was negatively associated with negative affect $(b=-0.032,95 \%[-0.038,-0.026], p<.001)$ and depressive/anxiety symptoms $(b=-0.010,95 \%$ CI $[-0.015,-0.004], p<.001)$, showing that participants reported reduced negative affect and depressive and anxiety symptoms over time.

\section{Effects of Education on Mental Health}


Results indicated that education was associated with the intercept but not the slope of eudaimonic well-being $(b=0.153,95 \%$ CI $[0.114,0.193], p<.001 ; b=-0.004,95 \%$ CI [-0.011, 0.003], $p=.21$, respectively). Similarly, education was associated with the intercept but not the slope of negative affect $(b=-0.081,95 \%$ CI $[-0.105,-0.058], p<.001 ; b=0.000,95 \%$ CI [$0.003,0.004], p=.82$, respectively) and of depressive and anxiety symptoms $(b=-0.066,95 \%$ CI [-0.087, -0.045], $p<.001 ; b=0.001,95 \%$ CI [-0.003, 0.004], $p=.60$, respectively). These results indicate that education was related to eudaimonic well-being, negative affect, and depressive and anxiety symptoms at baseline but was not associated with the rate of changes over time. Education, however, was not associated with neither intercept nor the slope of positive affect $(b=0.015,95 \%$ CI [-0.007, 0.036], $p=.18 ; b=-0.002,95 \%$ CI $[-0.005,0.002], p=.40$, respectively). The results remained very similar after controlling for gender, age, and selfisolation behavior (see Table 3).

\section{[INSERT TABLE 3 HERE]}

\section{The Mediation Effect of Financial Stress}

The MMM for eudaimonic well-being showed that education was associated with the intercept but not the slope of financial stress $(b=-0.120,95 \%$ CI $[-0.151,-0.089], p<.001 ; b=$ $0.000,95 \%$ CI [-0.005, 0.005], $p=.91$, respectively). Financial stress, in turn, was associated with eudaimonic well-being $(b=-0.199,95 \%$ CI $[-0.255,-0.143], p<.001)$. Education remained associated with the intercept but not the slope of eudaimonic well-being $(b=0.130,95 \% \mathrm{CI}$ $[0.090,0.170], p<.001 ; b=-0.004,95 \%$ CI $[-0.011,0.003], p=.21$, respectively). The results remained very similar after controlling for covariates (see Figure 1.1). There was a significant indirect effect of education on eudaimonic well-being through the intercept but not the slope of financial stress (effect $=0.025,95 \% \mathrm{CI}[0.016,0.035], p<.001$; effect $=0.000,95 \% \mathrm{CI}[-0.001$, 
0.001], $p=.86$, respectively), showing that there was a mediation effect of financial stress on the association between education and endaimonic well-being at baseline and that there were no temporal variations in the mediation effect of financial stress.

Similarly, the MMM for positive affect showed that education was associated with the intercept but not the slope of financial stress $(b=-0.120,95 \%$ CI $[-0.151,-0.089], p<.001 ; b=$ $0.000,95 \%$ CI $[-0.005,0.005], p=.90$, respectively). Financial stress was not associated with positive affect $(b=-0.016,95 \%$ CI $[-0.048,0.017], p=.34)$. Education remained not associated with the intercept or the slope of negative affect $(b=0.013,95 \%$ CI $[-0.009,0.035], p=.26 ; b=$ $-0.002,95 \% \mathrm{CI}[-0.005,0.002], p=.40$, respectively). The results were similar after adjusting for covariates (see Figure 1.2). There were no indirect effects of education on positive affect through neither the intercept nor the slope of financial stress (effect $=0.001,95 \% \mathrm{CI}[-0.003,0.005], p$ $=.59$; effect $=0.000,95 \% \mathrm{CI}[0.000,0.000], p=.85$, respectively $)$.

The MMM for negative affect indicated that education was associated with the intercept but not the slope of financial stress $(b=-0.120,95 \%$ CI $[-0.151,-0.089], p<.001 ; b=0.000$, $95 \%$ CI [-0.005, 0.005], $p=.90$, respectively). Financial stress, in turn, was associated with negative affect $(b=0.153,95 \%$ CI $[0.119,0.188], p<.001)$. Education remained associated with the intercept but not the slope of negative affect $(b=-0.063,95 \%$ CI $[-0.087,-0.039], p<.001 ; b$ $=0.000,95 \%$ CI $[-0.003,0.004], p=.81$, respectively). The results were similar after adjusting for covariates (see Figure 1.3), except that the association between education and the intercept of negative affect became statistically non-significant $(b=-0.020,95 \%$ CI $[-0.044,0.004], p=.11)$. There was a significant indirect effect of education on negative affect through the intercept but not the slope of financial stress $($ effect $=-0.019,95 \%$ CI $[-0.026,-0.013], p<.001$; effect $=$ $0.000,95 \% \mathrm{CI}[-0.001,0.001], p=.91$, respectively), showing that there was a mediation effect 
of financial stress on the association between education and negative affect at baseline and that there were no temporal variations in the mediation effect of financial stress.

The MMM for depressive and anxiety symptoms showed similar results. Education was related to the intercept but not the slope of financial stress $(b=-0.120,95 \%$ CI [-0.151, -0.089$]$, $p<.001 ; b=0.000,95 \%$ CI $[-0.005,0.005], p=.90$, respectively). Financial stress, in turn, was related to depressive and anxiety symptoms $(b=0.120,95 \%$ CI $[0.090,0.149], p<.001)$. Education remained related to the intercept but not the slope of negative affect $(b=-0.052,95 \%$ CI $[-0.073,-0.031], p<.001 ; b=0.001,95 \%$ CI $[-0.002,0.004], p=.59$, respectively). When including the covariates, the results remained similar (see Figure 1.4). The indirect effect of education on depressive and anxiety symptoms via the intercept but not slope of financial stress reached statistical significance $($ effect $=-0.015,95 \%$ CI $[-0.020,-0.009], p<.001$; effect $=0.000$, 95\%CI [-0.001, 0.001], $p=.85$, respectively), showing that there was a mediation effect of financial stress on the association between education and depressive and anxiety symptoms at baseline and that there were no temporal variations in the mediation effect of financial stress.

\section{[INSERT FIGURE 1 HERE]}

Some post hoc path analyses (i.e., these analyses were not pre-registered) were performed to further examine the temporal variations in the mediation effect of financial stress on the relationships between education and mental health outcomes. Specifically, we tested the mediation effect of financial stress at $\mathrm{T} 2$ on the associations between education and mental health outcomes (all mental health outcomes were included in the same model) assessed at T3 and $\mathrm{T} 4$, as well as the mediation effect of financial stress at $\mathrm{T} 3$ on the associations between education and mental health outcomes at T4. The significance of the indirect effect was examined using the bootstrapping method based on 1,000 resamples (MacKinnon, Lockwood, \& 
Williams, 2004). After controlling for age, gender, and self-isolation behavior at T2, results showed that education was associated with financial stress at T2 $(b=-0.119, p<.001)$, which, in turn, was associated with eudaimonic well-being $(b=-0.153, p<.001)$, negative affect $(b=$ $0.077, p<.001)$, and depressive and anxiety symptoms $(b=0.057, p=.003)$, but not positive affect at T3 $(b=-0.010, p=.65)$. Also, education was associated with financial stress at T2 $(b=-$ $0.119, p<.001)$, which, in turn, was associated with eudaimonic well-being $(b=-0.126, p$ $=.004)$, negative affect $(b=0.106, p<.001)$, and depressive and anxiety symptoms $(b=0.078, p$ $<.001)$, but not positive affect at $\mathrm{T} 4(b=-0.017, p=.51)$. Similarly, education was associated with financial stress at T3 $(b=-0.121, p<.001)$, which, in turn, was associated with eudaimonic well-being $(b=-0.134, p=.003)$, negative affect $(b=0.113, p<.001)$, and depressive and anxiety symptoms $(b=0.068, p=.002)$, but not positive affect at T4 $(b=0.002, p=.94)$. The indirect effects of education on mental health outcomes at $\mathrm{T} 3$ and at $\mathrm{T} 4$ via financial stress were all significant (see Table 4), with an exception that there were no significant indirect effects of education on positive affect at $\mathrm{T} 3$ and $\mathrm{T} 4$ via financial stress. These consistent results indicate the robust mediation effect of financial stress on the associations between education and mental health outcomes over time.

[INSERT TABLE 4 HERE]

\section{Discussion}

This study examined educational differences in trajectories of mental health outcomes at the early stages of the COVID-19 pandemic and the mediation effect of financial stress on the association between education and mental health. Consistent with our hypothesis, this study found that, overall, adults exhibited improvements in mental health outcomes, showing an increase in eudaimonic well-being and positive affect and a decline in negative affect and 
depressive and anxiety symptoms over time. As predicted, we found that there were educational disparities in eudaimonic well-being, negative affect, and depressive and anxiety symptoms at baseline, such that individuals with lower education reported lower levels of eudaimonic wellbeing but higher levels of negative affect and depressive and anxiety symptoms. Unexpectedly, however, such educational disparities remained stable over time, showing no observable educational differences in the rate of improvement in mental health outcomes. In addition, supporting our hypothesis regarding the mediation effect of financial stress, mediation analyses indicated that financial stress mediated the associations between education and eudaimonic wellbeing, negative affect, and depressive and anxiety symptoms at baseline, and there were no temporal variations in the mediation effects of financial stress.

The significant educational differences in mental health outcomes at baseline are in line with previous studies suggesting that low education is a risk factor for poor mental health at the beginning of the COVID-19 pandemic (Fancourt et al., 2021; Kola et al., 2021). This is not surprising, given that low education has been long recognized as a social determinant of poor health (Adler \& Ostrove, 1999). Interestingly, however, the present study found that such educational disparities in mental health remained stable, showing that individuals across different levels of education exhibited a similar rate of improvement in mental health outcomes during the initial months of the pandemic (between March and June 2020). These results are somewhat inconsistent with one previous study reporting a decline in educational disparities in mental health after the initial COVID-19 lockdown in the UK (between March and August 2020), which showed a faster decline in depression and anxiety among individuals with lower education (Fancourt et al., 2021). However, mixed findings in this emerging literature are not uncommon. For example, another study conducted during the pandemic in the UK documented a larger 
decline in mental health problems among those with a bachelor's degree than their counterpart without a bachelor's degree (Daly et al., 2020). The reasons for the inconsistent findings between existing studies and ours are unclear, particularly given that the longitudinal impact of the COVID-19 pandemic on mental health is still not fully understood. One possible explanation may be attributed to the differences in sample characteristics between existing studies (e.g., a more homogeneous sample of adults from the UK, Fancourt et al., 2021) and the present one (e.g., a more heterogeneous sample of adults from multiple countries). Future studies that involve participants from various countries are needed to verify this possible explanation. Nevertheless, our results highlight the persistent educational disparities in mental health outcomes at the early stages of the COVID-19 pandemic.

Consistent with our hypothesis, we found that financial stress mediated the association between education and mental health outcomes. That is, people who reported lower education reported experiencing greater financial stress, and when people experienced financial stress, they, in turn, reported poorer mental health. This result highlights the potential intermediate process of financial stress in contributing to the documented educational disparities in mental health in the context of the COVID-19 pandemic (Fancourt et al., 2021; Prati \& Mancini, 2021). Our results corroborate previous studies suggesting the disproportionate financial toll on those from low educational backgrounds during the COVID-19 pandemic (Kochhar, 2020; Perry, Aronson, \& Pescosolido, 2021). Moreover, our results did not find significant time interaction (i.e., effects of education on the slope of financial stress) in the mediation models for each mental health outcome, indicating no temporal variations in the mediation effects of financial stress. These results seem to suggest the relatively stable mediation process that financial stress played in contributing to persistent and relatively stable educational disparities in mental health 
outcomes during the study period. Altogether, these results are also in line with the larger literature suggesting the intermediate process of financial stress in contributing to socioeconomic disparities in health (Baum et al., 1999; Cundiff et al., 2020).

In addition, we observed overall improvements in mental health outcomes after the beginning of the COVID-19 pandemic in March 2020, showing increased eudaimonic well-being and positive affect and declined negative affect and depressive and anxiety symptoms over time. These results, together with findings from previous longitudinal studies (for a review, see Prati \& Mancini, 2021), highlight individuals' psychosocial resilience in response to the COVID-19 pandemic. However, evidence from the UK has suggested that although there were declines in mental health problems between April and June 2020, the level of mental health problems during the COVID-19 pandemic remains significantly higher than the pre-pandemic level (Daly et al., 2020). Notably, findings from previous studies, including ours, that focused on the impact of the COVID-19 pandemic on mental health are mainly based on data collected over a short period of time. It remains unknown whether the COVID-19 pandemic would lead to long-lasting effects on mental health.

A few limitations should be acknowledged when interpreting results in this study. First, the lack of pre-COVID-19 pandemic data on mental health outcomes may limit the interpretation of educational disparities in mental health. Our findings may not be able to shed light on whether the COVID-19 pandemic exacerbates the existing educational disparities in mental health, although initial evidence seems to suggest that it did (Perry et al., 2021). Second, this study included a relatively heterogeneous sample that consisted of participants from multiple countries. Individuals residing in different countries may have different experiences related to the COVID19 pandemic that they may be at different levels of risk of experiencing mental health problems. 
However, our analyses showed similar results after controlling for individuals' self-isolation behaviors. Also, evidence from a recent meta-analysis found no moderation effects of continent or days of lockdown on the impact of COVID-19 pandemic on mental health (Prati \& Mancini, 2021). Third, participants included in the current analysis were relatively well-educated, limiting the presentation at the lower end of the educational spectrum. Larger educational disparities may be evident in more representative samples. Despite these limitations, our study has several strengths. This study is among one of the few longitudinal studies to examine the trajectory of mental health at the early stages of the COVID-19 pandemic in a sample of adults from various countries. Moreover, this study is one of the few studies to investigate the mediational process through which socioeconomic disparities in mental health occur during the COVID-19 pandemic. In addition, our hypotheses and statistical analyses were pre-registered on the Open Science Framework.

\section{Conclusion}

The COVID-19 pandemic has negatively affected many people's mental health and has been particularly challenging for those with low levels of education. Our results indicate the persistent educational disparities in mental health at the early stages of the COVID-19 pandemic and highlight the role that financial stress has played in contributing to such educational disparities. These results suggest the need to address financial stress related to the pandemic to narrow mental health disparities among adults from low educational backgrounds. 


\section{References}

Adler, N. E., \& Ostrove, J. M. (1999). Socioeconomic status and health: What we know and what we don't. Annals of the New York Academy of Sciences, 896(1), 3-15.

Baum, A., Garofalo, J., \& Yali, A. M. (1999). Socioeconomic status and chronic stress: Does stress account for SES effects on health? Annals of the New York Academy of Sciences, 896(1), 131-144.

Berli, C., Inauen, J., Stadler, G., Scholz, U., \& Shrout, P. E. (2020). Understanding betweenperson interventions with time-intensive longitudinal outcome data: Longitudinal mediation analyses. Annals of Behavioral Medicine, Epub ahead of print.

Bueno-Notivol, J., Gracia-García, P., Olaya, B., Lasheras, I., López-Antón, R., \& Santabárbara, J. (2021). Prevalence of depression during the COVID-19 outbreak: A meta-analysis of community-based studies. International Journal of Clinical and Health Psychology, 21(1), 100196.

Center for Systems Science and Engineering, Johns Hopkins University (2021). COVID-19 Dashboard. Retrieved from https://coronavirus.jhu.edu/map.html.

Coibion, O., Gorodnichenko, Y., \& Weber, M. (2020). Labor markets during the COVID-19 crisis: A preliminary view (0898-2937). Retrieved from https://www.nber.org/papers/w27017.

Colina, S., Marrone, N., Ingram, M., \& Sánchez, D. (2017). Translation quality assessment in health research: A functionalist alternative to back-translation. Evaluation \& the Health Professions, 40(3), 267-293. 
Cooke, J. E., Eirich, R., Racine, N., \& Madigan, S. (2020). Prevalence of posttraumatic and general psychological stress during COVID-19: A rapid review and meta-analysis. Psychiatry Research, 292, 113347.

Crayne, M. P. (2020). The traumatic impact of job loss and job search in the aftermath of COVID-19. Psychological Trauma: Theory, Research, Practice, and Policy, 12(S1), S180.

Cundiff, J. M., Boylan, J. M., \& Muscatell, K. A. (2020). The Pathway From Social Status to Physical Health: Taking a Closer Look at Stress as a Mediator. Current Directions in Psychological Science, 29(2), 147-153.

Daly, M., Sutin, A. R., \& Robinson, E. (2020). Longitudinal changes in mental health and the COVID-19 pandemic: Evidence from the UK Household Longitudinal Study. Psychological Medicine, 1-10.

Diener, E., Emmons, R. A., Larsen, R. J., \& Griffin, S. (1985). The satisfaction with life scale. Journal of Personality Assessment, 49(1), 71-75.

Enders, C. K., \& Tofighi, D. (2007). Centering predictor variables in cross-sectional multilevel models: A new look at an old issue. Psychological Methods, 12(2), 121-138.

Fancourt, D., Steptoe, A., \& Bu, F. (2021). Trajectories of anxiety and depressive symptoms during enforced isolation due to COVID-19 in England: A longitudinal observational study. The Lancet Psychiatry, 8(2), 141-149.

Faria-e-Castro, M. (2021). Fiscal policy during a pandemic. Journal of Economic Dynamics and Control, 125, 104088. 
Gallo, L. C., \& Matthews, K. A. (2003). Understanding the association between socioeconomic status and physical health: do negative emotions play a role? Psychological Bulletin, $129(1), 10-51$

Hwang, T.-J., Rabheru, K., Peisah, C., Reichman, W., \& Ikeda, M. (2020). Loneliness and social isolation during the COVID-19 pandemic. International Psychogeriatrics, 32(10), 1217 1220.

Jiang, Y., Zilioli, S., Rodriguez-Stanley, J., Peek, K. M., \& Cutchin, M. P. (2020). Socioeconomic status and differential psychological and immune responses to a humancaused disaster. Brain, Behavior, and Immunity, 88, 935-939.

Kochhar, R. (2020). Unemployment rose higher in three months of COVID-19 than it did in two years of the Great Recession. Pew Research Center. Retrieved from https://www.pewresearch.org/fact-tank/2020/06/11/unemployment-rose-higher-in-threemonths-of-covid-19-than-it-did-in-two-years-of-the-great-recession/.

Kola, L., Kohrt, B. A., Hanlon, C., Naslund, J. A., Sikander, S., Balaji, M., . . Gonsalves, P. (2021). COVID-19 mental health impact and responses in low-income and middleincome countries: Reimagining global mental health. The Lancet Psychiatry. Epub ahead of print.

Larsen, R. (2011). Missing data imputation versus full information maximum likelihood with second-level dependencies. Structural Equation Modeling: A Multidisciplinary Journal, $18(4), 649-662$.

Löwe, B., Wahl, I., Rose, M., Spitzer, C., Glaesmer, H., Wingenfeld, K., . . Brähler, E. (2010). A 4-item measure of depression and anxiety: Validation and standardization of the 
Patient Health Questionnaire-4 (PHQ-4) in the general population. Journal of Affective Disorders, 122(1-2), 86-95.

MacKinnon, D. P., Lockwood, C. M., \& Williams, J. (2004). Confidence limits for the indirect effect: Distribution of the product and resampling methods. Multivariate Behavioral Research, 39(1), 99-128.

O'Connor, R. C., Wetherall, K., Cleare, S., McClelland, H., Melson, A. J., Niedzwiedz, C. L., .. . Scowcroft, E. (2020). Mental health and well-being during the COVID-19 pandemic: Longitudinal analyses of adults in the UK COVID-19 Mental Health \& Wellbeing study. The British Journal of Psychiatry, 1-8.

Okechukwu, C. A., El Ayadi, A. M., Tamers, S. L., Sabbath, E. L., \& Berkman, L. (2012). Household food insufficiency, financial strain, work-family spillover, and depressive symptoms in the working class: The work, family, and health network study. American Journal of Public Health, 102(1), 126-133.

Paleari, F. G., Pivetti, M., Galati, D., \& Fincham, F. D. (2021). Hedonic and eudaimonic wellbeing during the COVID-19 lockdown in Italy: The role of stigma and appraisals. British Journal of Health Psychology, 26(2), 657-678.

Pappas, S. (2020). How will people react to the new financial crisis. Washington, DC: American Psychological Association. Retrieved from https://www.apa.org/news/apa/2020/financial-

\section{crisis-covid-19.}

Parnia, A., \& Siddiqi, A. (2020). Socioeconomic disparities in smoking are partially explained by chronic financial stress: Marginal structural model of older US adults. Journal of Epidemiology Community Health, 74(3), 248-254. 
Pearlin, L. I., Menaghan, E. G., Lieberman, M. A., \& Mullan, J. T. (1981). The stress process. Journal of Health and Social behavior, 337-356.

Perry, B. L., Aronson, B., \& Pescosolido, B. A. (2021). Pandemic precarity: COVID-19 is exposing and exacerbating inequalities in the American heartland. Proceedings of the National Academy of Sciences, 118(8), e2020685118.

Prati, G., \& Mancini, A. (2021). The psychological impact of COVID-19 pandemic lockdowns: A review and meta-analysis of longitudinal studies and natural experiments. https://doi.org/10.1017/S0033291721000015.

Preacher, K. J., Zhang, Z., \& Zyphur, M. J. (2011). Alternative methods for assessing mediation in multilevel data: The advantages of multilevel SEM. Structural Equation Modeling, $18(2), 161-182$

Purtle, J. (2020). COVID-19 and mental health equity in the United States. Social Psychiatry and Psychiatric Epidemiology, 55(8), 969-971.

Rios, R., \& Zautra, A. J. (2011). Socioeconomic disparities in pain: The role of economic hardship and daily financial worry. Health Psychology, 30(1), 58-66.

Salari, N., Hosseinian-Far, A., Jalali, R., Vaisi-Raygani, A., Rasoulpoor, S., Mohammadi, M., .. Khaledi-Paveh, B. (2020). Prevalence of stress, anxiety, depression among the general population during the COVID-19 pandemic: A systematic review and metaanalysis. Globalization and Health, 16(1), 1-11.

Thayer, Z. M., \& Gildner, T. E. (2020). COVID-19-related financial stress associated with higher likelihood of depression among pregnant women living in the United States. American Journal of Human Biology, e23508. 
Tyupa, S. (2011). A theoretical framework for back-translation as a quality assessment tool. New Voices in Translation Studies, 7(1), 35-46.

Wang, C., Pan, R., Wan, X., Tan, Y., Xu, L., McIntyre, R. S., . . Sharma, V. K. (2020). A longitudinal study on the mental health of general population during the COVID-19 epidemic in China. Brain, Behavior, and Immunity, 87, 40-48.

Watson, D., Clark, L. A., \& Tellegen, A. (1988). Development and validation of brief measures of positive and negative affect: The PANAS scales. Journal of Personality and Social Psychology, 54(6), 1063-1070.

Wilson, J. M., Lee, J., Fitzgerald, H. N., Oosterhoff, B., Sevi, B., \& Shook, N. J. (2020). Job insecurity and financial concern during the COVID-19 pandemic are associated with worse mental health. Journal of Occupational and Environmental Medicine, 62(9), 686691. 
Table 1. Mean, standard deviation (SD), and range of study variables.

\begin{tabular}{|c|c|c|c|c|c|c|c|c|}
\hline \multirow[b]{3}{*}{ Variables } & \multicolumn{8}{|c|}{ Waves } \\
\hline & \multicolumn{2}{|c|}{ Baseline } & \multicolumn{2}{|c|}{ 2-week follow-up } & \multicolumn{2}{|c|}{ 4-week follow-up } & \multicolumn{2}{|c|}{ 6-week follow-up } \\
\hline & Mean (SD) & Range & Mean (SD) & Range & Mean (SD) & Range & Mean (SD) & Range \\
\hline Education & $5.84(1.51)$ & $1-8$ & - & - & - & - & - & - \\
\hline Female $(N, \%)$ & $1808(82.0)$ & - & - & - & - & - & - & - \\
\hline Other gender $(N, \%)$ & $47(2.1)$ & & & & & & & \\
\hline Age & $32.75(12.59)$ & $18-88$ & - & - & - & - & - & - \\
\hline Eudaimonic well-being & 4.01(1.43) & $1-7$ & $4.06(1.41)$ & $1-7$ & $4.14(1.42)$ & $1-7$ & $4.27(1.44)$ & $1-7$ \\
\hline Negative affect & $2.69(0.86)$ & $1-5$ & $2.62(0.85)$ & $1-5$ & $2.56(0.86)$ & $1-5$ & $2.43(0.84)$ & $1-5$ \\
\hline Positive affect & $2.57(0.78)$ & $1-5$ & $2.53(0.77)$ & $1-5$ & $2.57(0.79)$ & $1-5$ & $2.62(0.80)$ & $1-5$ \\
\hline DA symptoms & $2.04(0.75)$ & $1-4$ & $2.04(0.76)$ & $1-4$ & $2.02(0.75)$ & $1-4$ & $1.95(0.72)$ & $1-4$ \\
\hline Financial stress & $2.31(1.19)$ & $1-5$ & $2.27(1.15)$ & $1-5$ & $2.20(1.13)$ & $1-5$ & $2.15(1.11)$ & $1-5$ \\
\hline Self-isolation behavior & $4.39(0.76)$ & $1-4$ & $4.33(0.79)$ & $1-4$ & $4.11(0.91)$ & $1-4$ & $3.78(1.04)$ & $1-4$ \\
\hline
\end{tabular}

Note. $\mathrm{DA}=$ depressive and anxiety. 
Table 2. Bivariate correlation coefficients between study variables.

\begin{tabular}{|c|c|c|c|c|c|c|c|c|c|c|c|c|c|c|c|c|c|c|c|c|c|c|c|c|c|c|}
\hline & 1 & 2 & 3 & 4 & 5 & 6 & 7 & 8 & 9 & 10 & 11 & 12 & 13 & 14 & 15 & 16 & 17 & 18 & 19 & 20 & 21 & 22 & 23 & 24 & 25 & 26 \\
\hline 1. Education & - & $.32^{* * 2}$ & $.16^{* * \pi}$ & $.16^{* * * *}$ & $.14^{* * *}$ & $.16^{* * *}$ & $-.13^{* * z}$ & $-.16^{* * *}$ & $-.12^{* * *}$ & $-.12^{* * *}$ & .03 & .02 & .04 & .01 & $-.12^{* * * *}$ & $-.13^{* * 8}$ & $-.13^{* * *+}$ & $-.10^{* 3}$ & $-16^{3 * *+}$ & $-.14^{* * 5}$ & $-.15^{* * *}$ & $-.17^{* * *}$ & $-.05^{*}$ & -.05 & -.01 & $.10^{* * 4}$ \\
\hline 2. Age & & & $.21^{* * *}$ & $.18^{* * * 4}$ & $.15^{* * *}$ & $.15^{* * t}$ & $-.26^{* * 8}$ & $-.25^{* * *}$ & $-.25^{* * *}$ & $-.23^{*+4}$ & $.04^{*}$ & .03 & .05 & .05 & $-.21^{* * *}$ & $-.21^{* * *}$ & $-.26^{* * *}$ & $-.25^{* 3 s}$ & -.02 & -.01 & -.02 & -.03 & $-.09^{* * * *}$ & $-.10^{* * * *}$ & -.04 & .03 \\
\hline 3. T1 EW & & & & $.66^{* * *}$ & $.62^{* * *}$ & $.58^{* * z}$ & $-.53^{* * s}$ & $-48^{* * t}$ & $-44^{* * *}$ & $-44^{* 2=}$ & $54^{* * *}$ & $43^{* * *}$ & $39^{* * *}$ & $41^{* * *}$ & $-.55^{* * * *}$ & $-48^{8 * z}$ & $-43^{* * * t}$ & $-43^{* s s}$ & $-15^{* * t}$ & $-15^{* s t}$ & $-15^{* * * *}$ & $-12^{2 * n+4}$ & -.03 & -.04 & -.02 & .03 \\
\hline 4. T2 EW & & & & & $.70^{* * *}$ & $.66^{s * t}$ & $-.44^{* * s}$ & $-.57^{* * *}$ & $-.48^{* * * *}$ & $-.47^{* 28}$ & $.44^{* * *}$ & $55^{* * *}$ & $47^{* * *}$ & $41^{* * *}$ & $-45^{* * * *}$ & $-57^{78 z}$ & $-46^{* m a n}$ & $-43^{* * 8}$ & $-15^{* * t}$ & $-17^{* 8 s}$ & $-15^{* * 8}$ & $-11^{* * * *}$ & $-07^{* *}$ & $\begin{array}{l}-.04 \\
-09^{* * *+1}\end{array}$ & $\begin{array}{l}-.02 \\
-08^{* *}\end{array}$ & $\begin{array}{l}-.03 \\
-.07^{*}\end{array}$ \\
\hline 5. T3 EW & & & & & - & $.72^{* * *}$ & $-.41^{* * * 8}$ & $-.46^{* * 4}$ & $-.55^{* * * *}$ & $-.54^{* 04}$ & $.45^{* * * *}$ & $.49^{* * *}$ & $.60^{* * * *}$ & $.51^{* * * *}$ & $-.44^{* *+4}$ & $-.49^{* * s+}$ & $-.57^{* * * *}$ & $-.51^{* s s}$ & $-.15^{* * t}$ & $-14^{* s s}$ & $-.18^{* * *+}$ & $-.14^{* * * 4}$ & $-07^{* *}$ & $-06^{8}$ & $\begin{array}{l}-.00 \\
-.09^{*=3}\end{array}$ & $\begin{array}{l}-.07 \\
-12^{* * * *}\end{array}$ \\
\hline 6. T4 EW & & & & & & - & $-.39^{* * s}$ & $-.43^{* * *}$ & $-.45^{* * *}$ & $-.56^{* 28}$ & $40^{* * *}$ & $43^{* * * *}$ & $.52^{* * *}$ & $.61^{* * * *}$ & $-.41^{* * * *}$ & $-.45^{* * 8}$ & $-.50^{* * * *}$ & $-.59^{* s z}$ & $-.11^{* * * *}$ & $-.13^{* * * 8}$ & $-.13^{* * *}$ & $-.11^{s * *}$ & $-.06^{*}$ & $-.08^{*}$ & $-.12^{* * * 3}$ & $-13^{* * n+4}$ \\
\hline 7. T1 NA & & & & & & & - & $77^{* * 8}$ & $72^{* * *}$ & $65^{* * *}$ & $-41^{*+n+4}$ & $-33^{* * z 8}$ & $-30^{* * * *}$ & $-27^{* * 8 s}$ & $73^{* * z}$ & $60^{* * * *}$ & $57^{* * z}$ & $52^{* * *}$ & $21^{* * z}$ & $15^{* * *}$ & $15^{* * * *}$ & $17^{* * z}$ & $.07^{* *}$ & $.09^{* * z}$ & $13^{* * * *}$ & -13 \\
\hline $8 . \mathrm{T} 2 \mathrm{NA}$ & & & & & & & & $\because$ & $80^{* * *}$ & $75^{* * *}$ & $-38^{*+n+4}$ & $-42^{* * n}$ & $-37^{* * * *}$ & $-30^{* z=8}$ & $63^{* * 8}$ & $74^{* * * *}$ & $64^{* * z}$ & $56^{* * *}$ & $19^{* * z}$ & $18^{* * *}$ & $18^{* * * *}$ & $20^{* z 8}$ & $09^{* * *}$ & $13^{* * z}$ & $14^{* * *}$ & $.12 * 5^{* * 8}$ \\
\hline 9. T3 NA & & & & & & & & & & $.81^{* * *}$ & $-.39^{* * * *}$ & $-39^{* 5 s}$ & $-.42^{* * t}$ & $-.37^{* * 8}$ & $.61^{* * *}$ & $.64^{* * * *}$ & $.76^{* * * 8}$ & $.63^{* * * *}$ & $.17^{* * *}$ & $.13^{* * *}$ & $.17^{* * * *}$ & $.20^{*=8}$ & $.10^{* * * *}$ & $.15^{* * 8}$ & $.18^{* * * t}$ & $.19^{* * s}$ \\
\hline 10. T4 NA & & & & & & & & & & - & $-.30^{* * *}$ & $-.31^{3 * s}$ & $-.38^{* * t}$ & $-.42^{* * s}$ & $.57^{* * z}$ & $.60^{* * * *}$ & $.69^{* * z}$ & $.76^{* * * *}$ & $.18^{* * 3}$ & $.17^{* * *}$ & $.18^{* * *}$ & $.20^{* s *}$ & $.10^{* *}$ & $.11^{* 8}$ & $.18^{* * *}$ & $.22^{* * z}$ \\
\hline 11. T1 PA & & & & & & & & & & & & $.69^{* * * *}$ & $.66^{* * *}$ & $.60^{3+s+}$ & $-.47^{* * * 4}$ & $-.40^{* 3 *}$ & $-.38^{* * *}$ & $-.33^{ \pm * s}$ & $-.05^{*}$ & $-.05^{*}$ & -.02 & -.01 & -.04 & $-.08^{* *}$ & $-.12^{* * z}$ & $-14^{* * *}$ \\
\hline 12. T2 PA & & & & & & & & & & & & & $.73^{* * * *}$ & $.65^{* * *}$ & $-.39^{* * * *}$ & $-.48^{* * 3}$ & $-.42^{* * *}$ & $-.32^{2 * 38}$ & -.03 & $-.05^{*}$ & -.05 & -.01 & $-.08^{* * * 4}$ & $-.13^{* * * *}$ & $-.14^{* * z}$ & $-.14^{* * * *}$ \\
\hline 13. T3 PA & & & & & & & & & & & & & & $.76^{* * *}$ & $-.37^{* * * *}$ & $-.41^{* * * 8}$ & $-.50^{* * * *}$ & $-.40^{* s 8}$ & -.02 & -.03 & -.02 & -.01 & $-.09^{* z}$ & $-.12^{* * * *}$ & $-.16^{* * z}$ & $-.19^{* * *}$ \\
\hline 14. T4 PA & & & & & & & & & & & & & & & $-.32^{* * * *}$ & $-.35^{* * * *}$ & $-42^{* * *}$ & $-.47^{* 38 s}$ & .03 & -.04 & -.02 & .01 & $-10^{* * z}$ & $-.14^{* * * *}$ & $-16^{* * z}$ & $-.19^{* * *}$ \\
\hline 15. T1 DAS & & & & & & & & & & & & & & & & $.72^{* * * *}$ & $.67^{* * 5}$ & $.62^{* * *}$ & $.20^{* * 8}$ & $.14^{* * * *}$ & $.14^{* * * *}$ & $13^{* 8 s}$ & $.12^{* * * *}$ & $.13^{* s 8}$ & $.14^{* * *}$ & $.14^{* * 8}$ \\
\hline 16. T2 DAS & & & & & & & & & & & & & & & & & $.73^{* * 8}$ & $.64^{* * * *}$ & $.17^{* * 8}$ & $.17^{* * *}$ & $.15^{* * *}$ & $14^{* z=8}$ & $.13^{* * * *}$ & $.15^{* * 8}$ & $15^{* * * t}$ & $.15^{* * 8}$ \\
\hline 17. T3 DAS & & & & & & & & & & & & & & & & & & $.74^{* * * *}$ & $.14^{* * *}$ & $.11^{* * *}$ & $.14^{* * * *}$ & $.14^{* * s}$ & $.14^{* * * *}$ & $.15^{* * * 8}$ & $17^{* * * *}$ & $19^{* z s}$ \\
\hline 18. T4 DAS & & & & & & & & & & & & & & & & & & & $.13^{* * 8}$ & $.15^{* * *}$ & $.14^{* * * *}$ & $.16^{* * 88}$ & $.13^{* * *}$ & $.14^{* \neq z 8}$ & $.18^{* * * t}$ & $21^{* z 8}$ \\
\hline 19. T1 FS & & & & & & & & & & & & & & & & & & & - & $.73^{* * *}$ & $.71^{* * * *}$ & $\begin{array}{l}.109^{* * s} \\
\text { s. }\end{array}$ & $.08^{* * * *}$ & $.06^{* * 8}$ & $.08^{* *}$ & .03 \\
\hline 20. T2 FS & & & & & & & & & & & & & & & & & & & & & $.77^{* * *}$ & $.75^{* s=8}$ & $.07^{* *}$ & $.08^{* * * *}$ & $.10^{* * *}$ & $.11^{* * *}$ \\
\hline 21. T3 FS & & & & & & & & & & & & & & & & & & & & & & $.79^{* 28}$ & $.06^{*}$ & $.10^{* *}$ & $12^{* * *}$ & $.11^{* * z}$ \\
\hline 22. T4 FS & & & & & & & & & & & & & & & & & & & & & & & .04 & $.11^{* *}$ & $.10^{* * *}$ & $.12^{* * *}$ \\
\hline 23. T1 SI & & & & & & & & & & & & & & & & & & & & & & & 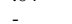 & $59^{* z *}$ & $50^{* * *}$ & $\begin{array}{l}.12=0 \\
41^{* * * 8}\end{array}$ \\
\hline $24 . \mathrm{T} 2 \mathrm{SI}$ & & & & & & & & & & & & & & & & & & & & & & & & & $63^{* * * *}$ & $.412^{* * z}$ \\
\hline 25. T3 SI & & & & & & & & & & & & & & & & & & & & & & & & & & $.68^{* * *}$ \\
\hline 26. T4 SI & & & & & & & & & & & & & & & & & & & & & & & & & & \\
\hline
\end{tabular}

Note. T1 = baseline; T2 = 2-week follow-up; T3= 4-week follow-up; T4= 6-week follow-up; EW = eudaimonic well-being; NA = negative affect; $\mathrm{PA}=$ positive affect $; \mathrm{DAS}=$ depressive and anxiety symptoms; $\mathrm{FS}=$ financial stress; $\mathrm{SI}=$ self-isolation behavior. ${ }^{*} p<.05 ;{ }^{* *} p<.01 ;{ }^{* * * *} p<.001$. 
Table 3. Effect of education on mental health.

\begin{tabular}{|c|c|c|c|c|c|c|c|c|}
\hline & \multicolumn{8}{|c|}{ Mental health outcomes } \\
\hline & \multicolumn{2}{|c|}{ Eudaimonic well-being } & \multicolumn{2}{|c|}{ Positive affect } & \multicolumn{2}{|c|}{ Negative affect } & \multicolumn{2}{|c|}{$\begin{array}{l}\text { Depressive and anxiety } \\
\text { symptoms }\end{array}$} \\
\hline & Estimate & $95 \% \mathrm{CI}$ & Estimate & $95 \% \mathrm{CI}$ & Estimate & $95 \% \mathrm{CI}$ & Estimate & $95 \% \mathrm{CI}$ \\
\hline \multicolumn{9}{|c|}{ Baseline outcome (intercept), $\pi_{0}$} \\
\hline Average intercept, $\beta_{00}$ & 4.484 & $(4.252,4.715)^{* * *}$ & 2.925 & $(2.797,3.054)^{* * *}$ & 2.179 & $(2.061,2.297)^{* * *}$ & 1.589 & $(1.478,1.699)^{* * *}$ \\
\hline Education, $\beta_{01}$ & 0.100 & $(0.059,0.142)^{* * *}$ & 0.009 & $(-0.013,0.032)$ & -0.039 & $(-0.063,-0.015)^{* *}$ & -0.037 & $(-0.058,-0.015)^{* *}$ \\
\hline Female, $\beta_{02}$ & -0.018 & $(-0.181,0.145)$ & -0.110 & $(-0.202,-0.018)^{*}$ & 0.187 & $(0.096,0.277)^{* * *}$ & 0.151 & $(0.068,0.233)^{* * *}$ \\
\hline Other gender, $\beta_{03}$ & -0.567 & $(-1.042,-0.093)^{*}$ & -0.348 & $(-0.578,-0.117)^{* *}$ & 0.598 & $(0.343,0.854)^{* * *}$ & 0.585 & $(0.343,0.827)^{* * *}$ \\
\hline Age, $\beta_{04}$ & 0.019 & $(0.014,0.024)^{* * *}$ & 0.002 & $(-0.001,0.004)$ & -0.016 & $(-0.018,-0.013)^{* * * *}$ & -0.010 & $(-0.013,-0.008)^{* * * *}$ \\
\hline \multicolumn{9}{|c|}{ Change in outcome (slope), $\pi_{1}$} \\
\hline Average slope, $\beta_{10}$ & 0.028 & $(0.002,0.053)^{*}$ & -0.007 & $(-0.021,0.008)$ & -0.013 & $(-0.026,0.001)$ & 0.003 & $(-0.009,0.016)$ \\
\hline Education, $\beta_{11}$ & -0.001 & $(-0.008,0.007)$ & -0.002 & $(-0.006,0.002)$ & -0.001 & $(-0.005,0.003)$ & 0.002 & $(-0.002,0.005)$ \\
\hline Female, $\beta_{12}$ & -0.001 & $(-0.029,0.027)$ & 0.013 & $(-0.003,0.028)$ & -0.016 & $(-0.031,-0.001)^{*}$ & -0.008 & $(-0.021,0.006)$ \\
\hline Other gender, $\beta_{13}$ & -0.046 & $(-0.144,0.051)$ & -0.011 & $(-0.053,0.030)$ & 0.017 & $(-0.029,0.063)$ & -0.003 & $(-0.051,0.045)$ \\
\hline Age, $\beta_{14}$ & -0.001 & $(-0.002,-0.000)^{*}$ & 0.000 & $(0.000,0.001)$ & 0.000 & $(0.000,0.001)$ & 0.000 & $(-0.001,-0.000)^{*}$ \\
\hline Self-isolation behavior, & & & & & & & & \\
\hline Intercept, $\beta_{20}$ & -0.100 & $(-0.139,-0.060)^{* * *}$ & -0.063 & $(-0.085,-0.040)^{* * *}$ & 0.077 & $(0.057,0.097)^{* * *}$ & 0.072 & $(0.054,0.091)^{* * *}$ \\
\hline
\end{tabular}


Table 4. Indirect effects of education on mental health via financial stress from path analyses.

\begin{tabular}{|c|c|c|}
\hline Indirect effect & Estimate & $95 \% \mathrm{CI}$ \\
\hline T1 Education $\rightarrow$ T2 Financial stress $\rightarrow$ T3 Eudaimonic Well-being & 0.018 & $(0.009,0.029)$ \\
\hline T1 Education $\rightarrow$ T2 Financial stress $\rightarrow$ T4 Eudaimonic Well-being & 0.015 & $(0.005,0.029)$ \\
\hline T1 Education $\rightarrow$ T3 Financial stress $\rightarrow$ T4 Eudaimonic Well-being & 0.016 & $(0.006,0.031)$ \\
\hline T1 Education $\rightarrow$ T2 Financial stress $\rightarrow$ T3 Positive affect & 0.001 & $(-0.004,0.006)$ \\
\hline T1 Education $\rightarrow$ T2 Financial stress $\rightarrow$ T4 Positive affect & 0.002 & $(-0.004,0.008)$ \\
\hline T1 Education $\rightarrow$ T3 Financial stress $\rightarrow$ T4 Positive affect & 0.000 & $(-0.006,0.006)$ \\
\hline T1 Education $\rightarrow$ T2 Financial stress $\rightarrow$ T3 Negative affect & -0.009 & $(-0.015,-0.004)$ \\
\hline T1 Education $\rightarrow$ T2 Financial stress $\rightarrow$ T4 Negative affect & -0.013 & $(-0.021,-0.007)$ \\
\hline T1 Education $\rightarrow$ T3 Financial stress $\rightarrow$ T4 Negative affect & -0.014 & $(-0.024,-0.007)$ \\
\hline T1 Education $\rightarrow$ T2 Financial stress $\rightarrow$ T3 Depressive and anxiety symptoms & -0.007 & $(-0.012,-0.003)$ \\
\hline T1 Education $\rightarrow$ T2 Financial stress $\rightarrow$ T4 Depressive and anxiety symptoms & -0.009 & $(-0.016,-0.004)$ \\
\hline T1 Education $\rightarrow$ T3 Financial stress $\rightarrow$ T4 Depressive and anxiety symptoms & -0.008 & $(-0.016,-0.003)$ \\
\hline
\end{tabular}

Note. $\mathrm{T} 1=$ baseline; $\mathrm{T} 2$ = 2-week follow-up; T3= 4-week follow-up; T4=6-week follow-up. 


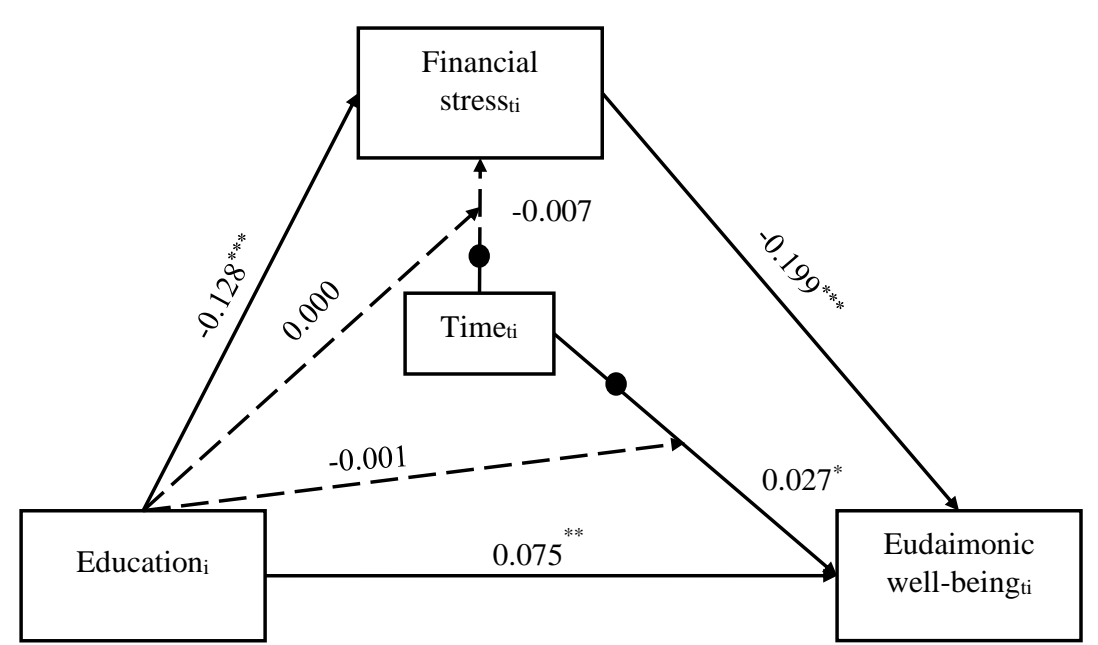

Figure 1.1. Multilevel mediation model of financial stress on the association between education and eudaimonic well-being.

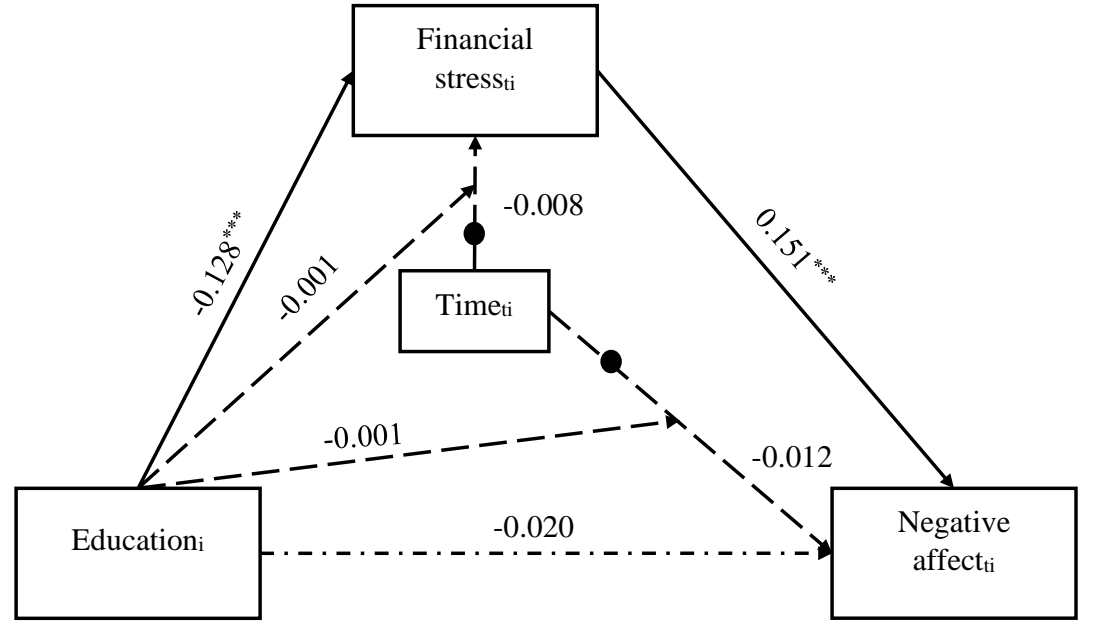

Figure 1.3. Multilevel mediation model of financial stress on the association between education and negative affect.

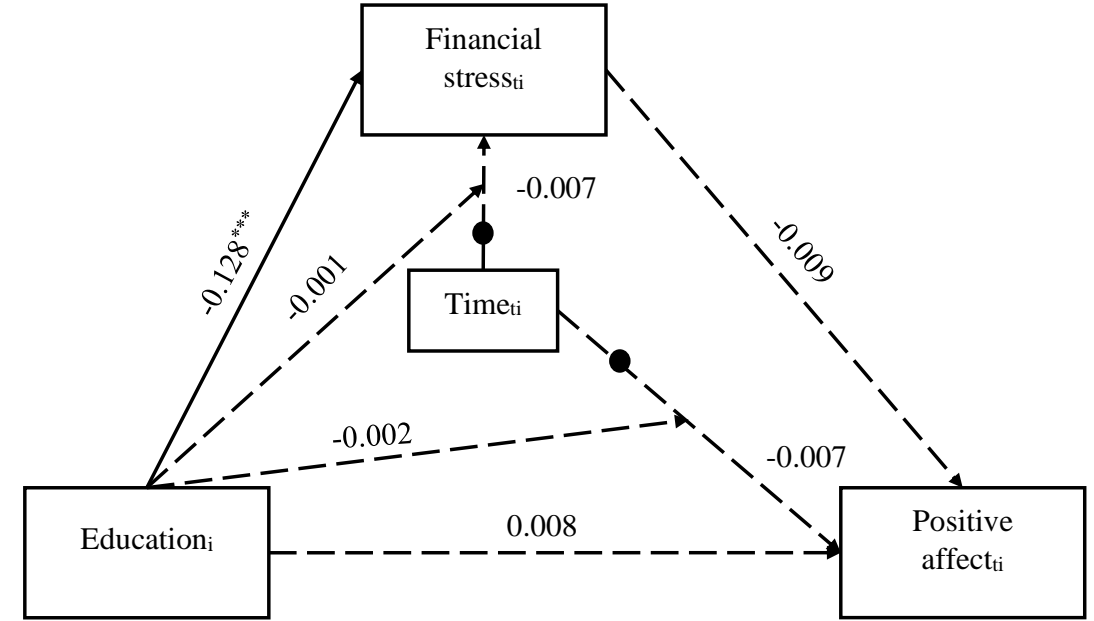

Figure 1.2. Multilevel mediation model of financial stress on the association between education and positive affect.

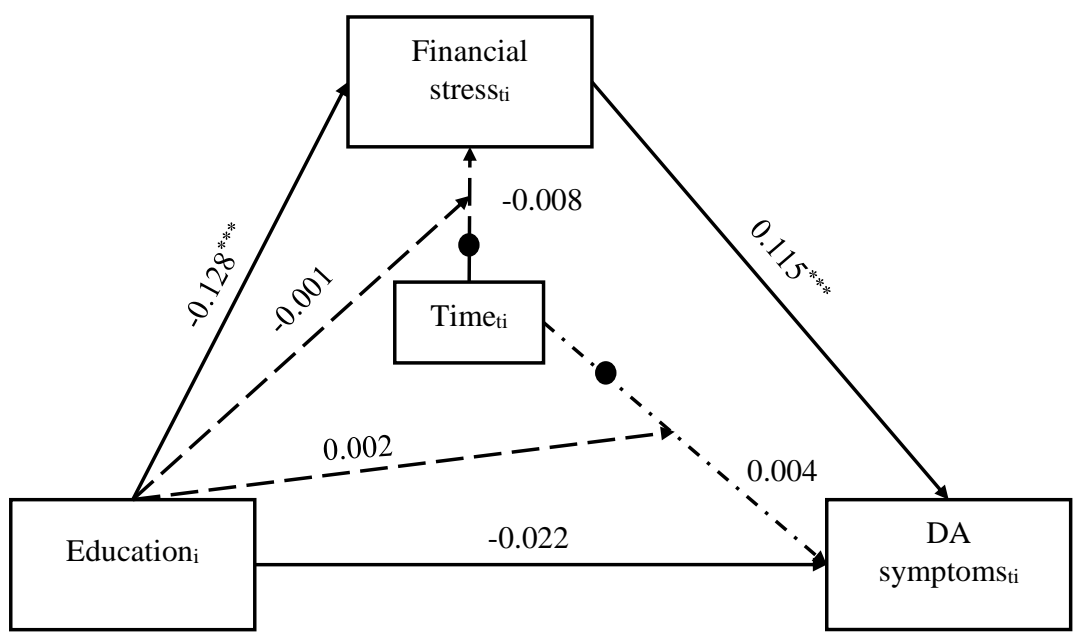

Figure 1.4. Multilevel mediation model of financial stress on the association between education and depressive symptoms.

Model 1. Multilevel mediation model of financial stress on the associations between education and mental health outcomes. Black dots presented random slopes; dashed lines represented statistical nonsignificance at $p>.05 ; \mathrm{i}=$ individual; $\mathrm{t}=$ time; $\mathrm{DA}=\mathrm{depressive}$ and anxiety. ${ }^{*} p<.05 ;{ }^{* *} p<.01{ }^{* * *} p<.001$. 